\title{
Static Detection of Thin Layers into Concrete with Ground Penetrating Radar
}

\author{
A. Van der Wielen ${ }^{1 *}$, L. Courard ${ }^{2}$ and F. Nguyen ${ }^{2}$ \\ ${ }^{1}$ FNRS Research Fellow, University of Liege, Belgium, e-mail: avdwielen@ulg.ac.be \\ ${ }^{2}$ University of Liege, Belgium \\ ${ }^{*}$ Corresponding author: A. Van der Wielen, E-mail: avdwielen@ulg.ac.be
}

\begin{abstract}
Ground Penetrating Radar (GPR) is a nondestructive technique particularly well adapted to the inspection of concrete structures and can help to determine the structure inner geometry or to detect damaged areas. When the GPR is used on structures containing thin layers, for example the sealing layer of a concrete bridge deck or the void into a masonry wall, it is important for the radar user to know the minimum thickness required to detect and estimate the thickness of those layers.

The theory of thin layer detection is based on a sine wave but, in reality, most commercial GPR systems emit a large frequency band wavelet, which undergoes attenuation into the layer. To analyze the influence of those realistic conditions on the reflection coefficient of a thin layer, we combined experimental measurements and numerical FDTD simulations. The experimental results matched the numerical predictions well, presenting a fast attenuation compared to the theoretical predictions. Nevertheless, for thicknesses inferior to $\lambda / 11$, the reflection coefficient could still be considered as linearly dependent of the thickness to wavelength ratio.
\end{abstract}

Keywords: GPR; Radar; Thin layer; NDT; Reflection. 


\section{Introduction}

Concrete structures can be attacked by diverse deterioration mechanisms, such as carbonation, alkali-aggregate reactions or corrosion. For this reason, it is important to frequently inspect the structures in order to be able to repair the damaged area and stop the degradation mechanisms. During the inspections, non-destructive techniques are particularly interesting because they permit to test large surfaces and they highly limit the number of cores that have to be extracted from the structure for laboratory tests. Among all the non-destructive methods, the Ground Penetrating Radar (GPR) is well adapted to inspect multilayer structures because only a percentage of the energy is reflected at every interface. An inspection can thus reveal the thickness and electromagnetic properties of two layers or more, if the conditions are favorable [1].

When thin layers are inspected, those characteristics cannot be determined straightforwardly anymore, because the incident radar waves (I) undergo multiple reflections on the two limit interfaces, which generates interferences. In this study, we tried to evaluate numerically and experimentally the minimum thickness that could be detected by the GPR.

\section{Principle of Ground Penetrating Radar}

\subsection{General Principle}

The principle of GPR is to send, with an emitter antenna, very short electromagnetic pulses through the ground or the structure. Those pulses propagate to all directions (or in the half-space downward only, if the antenna is shielded) and are reflected when they meet interfaces between media of different electromagnetic properties. A receiver antenna measures the amplitude of the signal over time (Fig. 1). On the resulting scan, both the direct wave and the waves reflected on the different interfaces can be observed. This method allows detecting voids, humid zones and material modifications.

\subsection{Radar Wave Speed Estimation}

If the conductivity of the material can be neglected (which is often the case for usual building materials [2]), the wave speed $v$ is directly dependant of the relative dielectric permittivity of the material $\varepsilon^{\prime}{ }_{r}$ (with $c$ the speed of light, equal to $30 \mathrm{~cm} / \mathrm{ns}$ ):

$$
v=\frac{c}{\sqrt{\varepsilon_{r}^{\prime}}}
$$

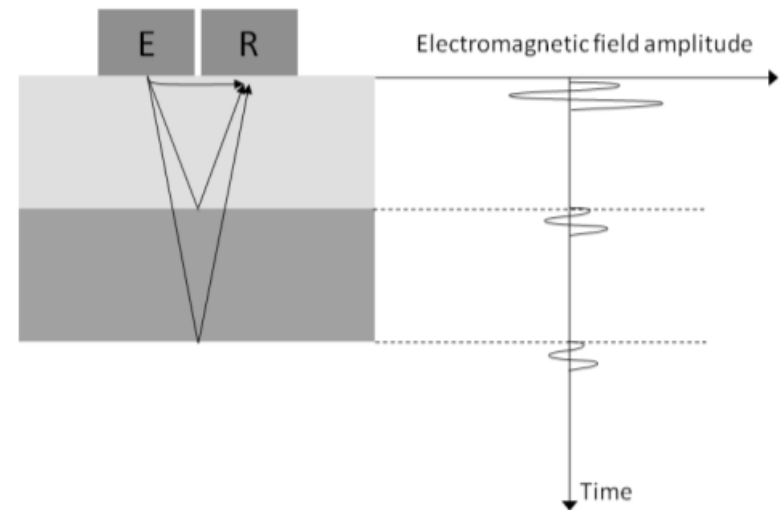

Figure 1: Simplified GPR signal measured on a two-layer structure.

Different methods are available to estimate the speed and thus the dielectric permittivity of concrete:

- By measuring the time elapsed between the emission of the pulse and the reception of its echo, the depth of the interface can be determined if the speed of the waves is known, and vice-versa.

- The measurement can be directly performed with two antennas (common mid-point measurement). The speed is then the slope of the time-versus-distance line representing the direct wave.

- The speed can also be estimated from the shape of the hyperbolas resulting from a punctual defect's detection.

- The dielectric permittivity can be estimated by using the reflection properties of the surface.

This last method is based on the reflection coefficient, which is the proportion of the incident wave reflected by an interface. It depends on the permittivities of the two media, as well as on the incident angle:

$$
R_{12}=\frac{\cos \theta_{1}-\sqrt{\frac{\varepsilon_{r 2}}{\varepsilon_{r 1}}-\sin ^{2} \theta_{1}}}{\cos \theta_{1}+\sqrt{\frac{\varepsilon_{r 2}}{\varepsilon_{r 1}}-\sin ^{2} \theta_{1}}}
$$

In eq. (2), derived from the Fresnel reflection coefficients [1], $\varepsilon_{r 1}$ and $\varepsilon_{r 2}$ are the respective relative dielectric permittivities of the two materials and $\theta_{1}$ is the inclination of the incident ray (Fig. 2 (a)).

If we measure the reflection amplitude of the surface from a certain distance, $\varepsilon_{r 1}=1$ (material 1 is air) and the inclination can be approximated to zero. 


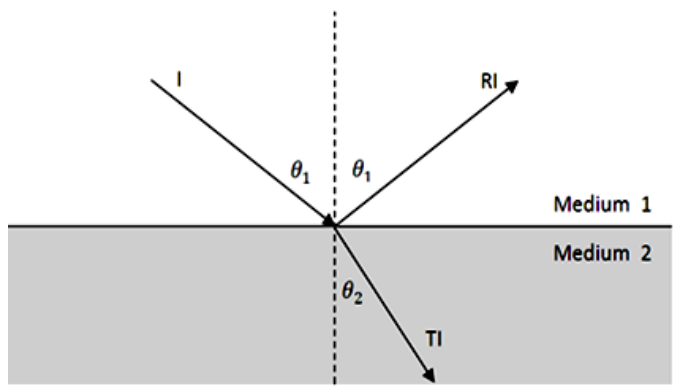

a

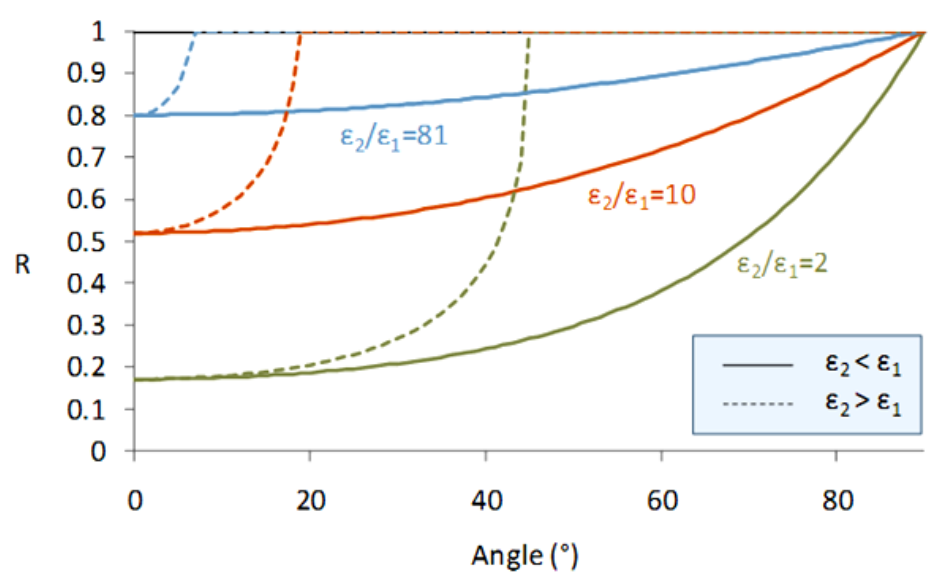

b

Figure 2: (a) The Fresnel reflection coefficient $(R)$ describes the proportion of the incident amplitude reflected at an interface; (b) Dependence of the reflection coefficient on the incident angle and permittivity contrast.

The reflection coefficient is obtained by dividing the reflected amplitude by the amplitude measured from the same distance on a perfect reflector; it thus univocally determines the dielectric permittivity of the concrete surface, from which it is possible to calculate the speed in the material. The drawback of this method is the fact that it only reveals the permittivity of the surface. If a gradient is present, the mean permittivity will be different from the surface permittivity, and the depth of the detected features could then be misevaluated.

When the tested material is loose, like sand or soil, a direct method can be used to estimate the dielectric properties, instead of the indirect techniques listed above: the TDR (Time Domain Reflectometer) method. TDR sensors are made of two metallic electrodes that allow a direct evaluation of the material's dielectric permittivity but have to be introduced into the material. For this reason, they are almost never used for concrete evaluation.

\section{Detection Limits of Thin Layers}

In many concrete structures, features presenting a small thickness to surface ratio are relatively common, i.e. the sealing layer of a bridge or the void into a masonry wall. It is thus important for the radar user to know the minimum thicknesses required to detect thin layers or to estimate their thicknesses.

When radar incident waves (I) encounter a thin layer, they undergo multiple reflections on the two limit interfaces, which generates constructive and destructive interferences (Fig. 3).

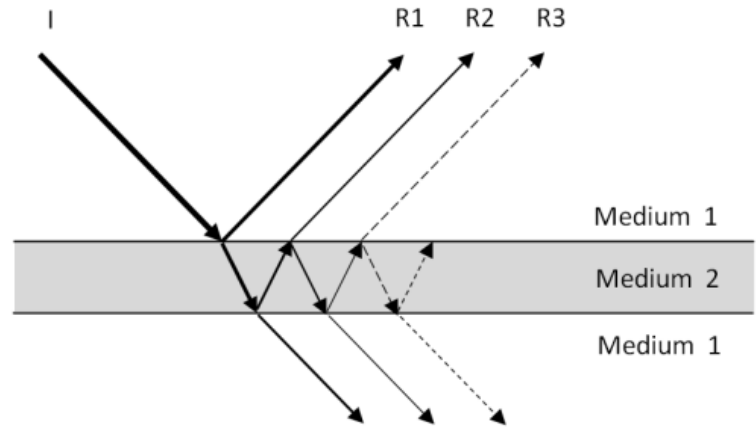

Figure 3: Multiple reflections in a thin layer.

The detection of the thin layer simply requires the measured amplitude to be higher than the noise level. It depends thus on the reflection coefficient, on the attenuation into the matrix (resulting from its conductivity [2]) and on the antenna-ground coupling, but also on the background noise level.

The resolution of the radar for thin layer detection is the minimum thickness required to be able to estimate the thickness of the layer. With a basic approach of a radargram inspection in the time-domain, the resolution corresponds then to the minimum thickness for which the first reflection on the two interfaces limiting the layer can be distinguished from one another. This happens when the traveltime through the layer is equal or larger than the pulse duration [2]. In the case of shielded, ground-coupled radars, the pulse duration is often comprised between two and three wave periods, due to the different positive and negative lobes present in the wavelet (Fig. 4). 


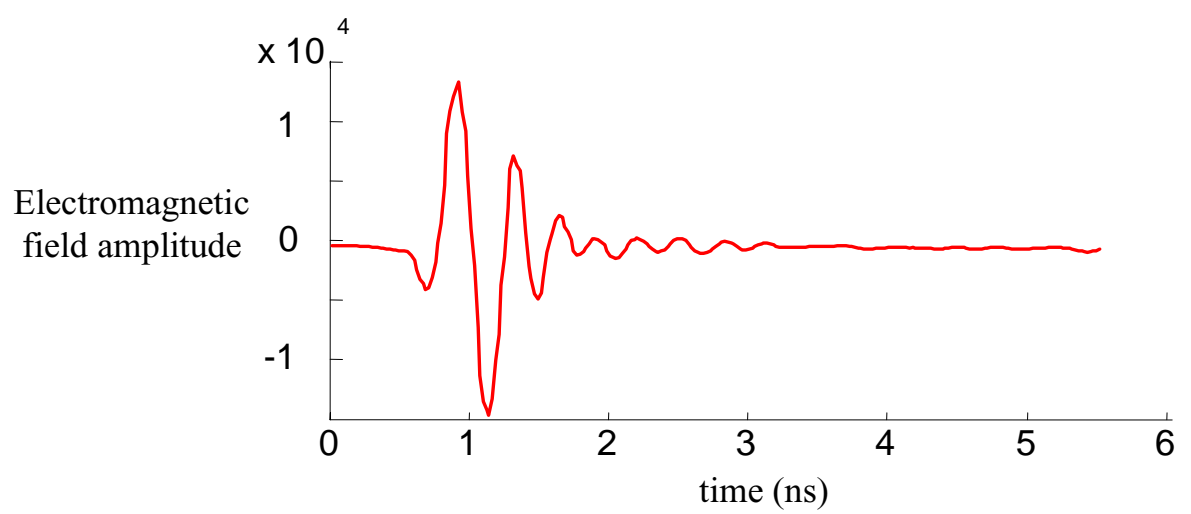

Figure 4: The $2.3 \mathrm{GHz}$ commercial radar incident wavelet measured in air presents several positive and negative lobes.

This relatively long pulse duration results in a rather low resolution, lower than the wavelength of the wave. When the layer is thinner, the different reflected wavelets cannot be distinguished in the radargram anymore: the shape of the resulting wavelet gets closer to that of its derivative, while its amplitude decreases [2,3]. But if the user knows that he is inspecting a thin layer, further information can be extracted from the radargram through the use of more sophisticated methods than a simple visual inspection. A global waveform inversion gives very good and accurate results $[4,5]$, but requires a detailed analysis of the radiating antenna. We propose here a simplified method, uniquely based on the peak-to-peak amplitude, which can be easily extracted from the radargram.

\subsection{Theoretical Approach}

The exact equation for a reflection from a single embedded layer and considering simple harmonic (sinusoidal) waves for a lossless layer was reported by Rayleigh [6]. The theoretical reflection coefficient is then given by eq. (3):

$$
R_{121}\left(\frac{d}{\lambda}, R_{12}\right)=\left|\frac{1-e^{\frac{i 4 \pi d}{\lambda}}}{1-R_{12}^{2} e^{\frac{i 4 \pi d}{\lambda}}} R_{12}\right|
$$

In eq. (3), $R_{121}$ is the reflection coefficient for the thin layer, $R_{12}$ is the coefficient corresponding to the reflection on a simple interface between the two media (2), $d$ is the layer thickness and $\lambda$ is the wavelength into the thin layer material.

The first maximum occurs when the layer thickness is equal to a quarter of the wavelength in the layer $(\lambda)$, and has then a periodicity of $\lambda / 2$. At the different maxima, the reflection occurring on the thin layer has a greater amplitude $R_{121, \text { max }}$ than a reflection $R_{12}$ that would occur on a simple interface. This amplitude is given by eq. (4):

$$
R_{121, \max }=\frac{2 R_{12}}{1+R_{12}^{2}}
$$

The evolution of $R_{121}$ with the ratio of the layer thickness on the wavelength in the thin layer is drawn in Fig. 5.

When the thickness of the layer can be considered as low compared to the wavelength (ratio up to 0.09 ), the reflection coefficient becomes directly proportional to the layer thickness $[2,3]$.

\subsection{Numerical Evaluation of the Thin Layer Reflection Coefficient}

We performed two different sets of simulations, with the finite difference time domain program GprMax2D [7]. In the first set, the incident wave was a continuous sine, in order to match the theoretical results and estimate the influence of the attenuation into the layer. Then, in the second set, we introduced a realistic pulse to predict experimental results.

The first simulations, performed with a continuous sinusoidal incident wave $(2.3 \mathrm{GHz})$, were carried out for an air layer placed at a depth of $10 \mathrm{~cm}$ into concrete $\left(\varepsilon_{1} / \varepsilon_{2}=10\right)$ and for a $4 \mathrm{~cm}$ antenna separation. The reflection coefficient was obtained by comparing the maximum peak-to-peak amplitude (after suppression of the direct wave) to the amplitude of a wave reflected on a perfect reflector. With this method, the contributions of the geometric and intrinsic attenuations into the concrete are suppressed, as well as the radiation pattern effects, because they are exactly the same in both configurations. Those parameters decrease globally the measured amplitude, but are independent of the reflection coefficient. 


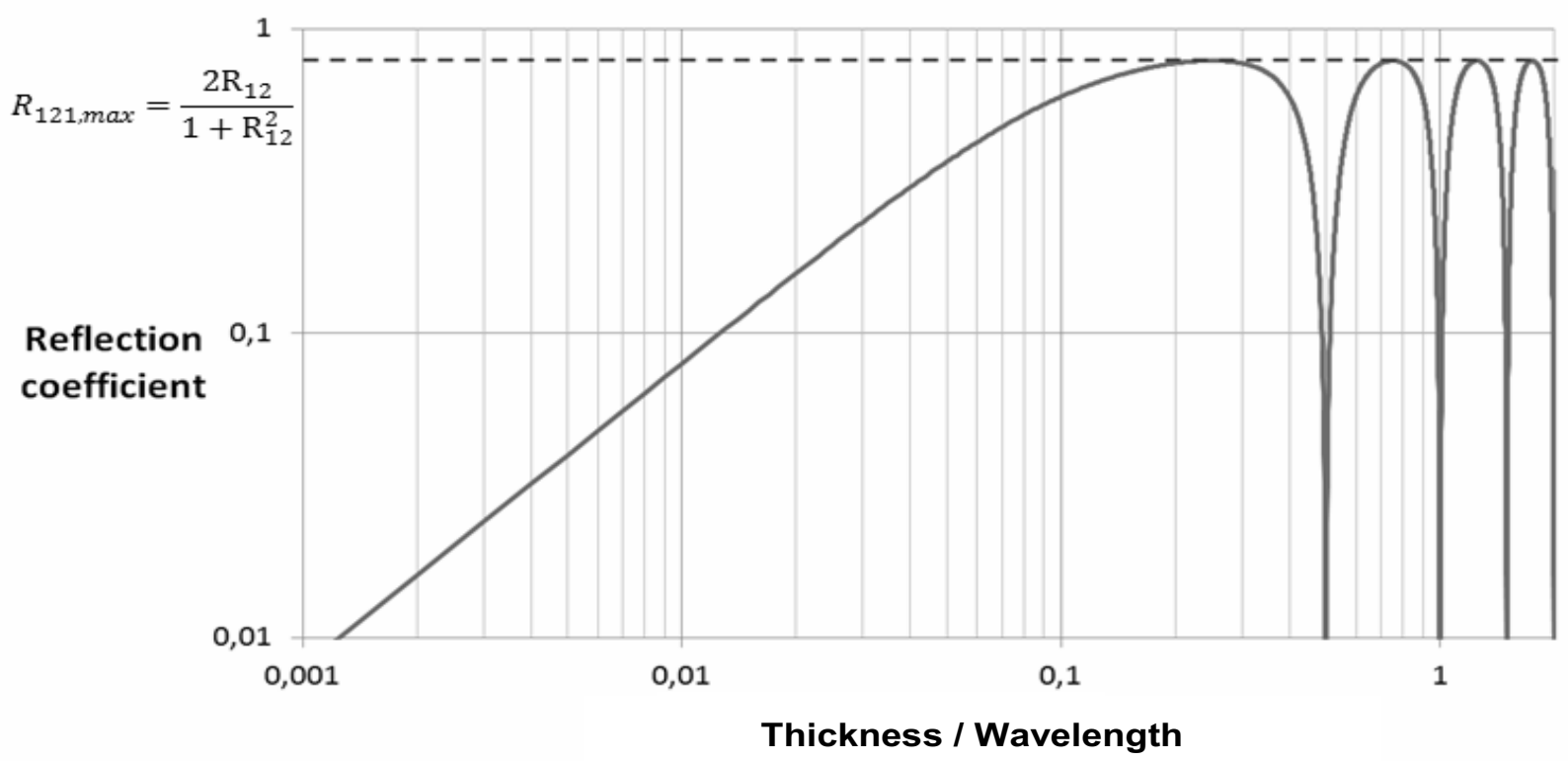

Figure 5: Reflection coefficient for an air thin layer into concrete.

Two parameters that weren't corrected are the geometric attenuation into the thin layer (which is different for each ray, depending on the number of reflections) and the fact that the simulated wave is not plane but cylindrical, due to the proximity and frequency of the source. The numerical results, displayed in Fig. 6, appeared to be in a relatively good concordance with the analytical predictions, but tend to stabilize due to the geometric attenuation into the layer.

A second set of simulations was carried out, in order to predict the reflection coefficient that would be measured with a pulse ground penetrating radar, more frequently used for concrete structures inspections. A realistic pulse derived from measurements performed with our radar (a Mala 2.3 $\mathrm{GHz}$ antenna) was thus introduced into GprMax2D, instead of a continuous sine. The results are displayed in Fig. 6 as well. The reflection coefficient of the realistic pulse is rapidly attenuated, because the two wavelets get separated from each other. Nevertheless, we can observe that for layers thinner than $\lambda / 11$ (for an error inferior to $10 \%$ ), the reflection coefficient still depends linearly on the thickness to wavelength ratio. It can then be estimated by the following linear approximation eq. (5) [6]:

$$
R_{121} \cong 4 \pi \frac{R_{12}}{1-R_{12}^{2}} \frac{d}{\lambda}
$$

\subsection{Experimental Measurement of the Thin Layer Reflection Coefficient}

A first experimental study was performed on two smooth, $10 \mathrm{~cm}$ thick concrete slabs. Between those slabs, a variable air gap was introduced (Fig. 7 (a)) through the use of plastic or wooden spacers of variable thicknesses (ranging from 1 to $96 \mathrm{~mm}$ ). As for the computations, the direct wave was subtracted from the resulting signal, and each amplitude was compared to the amplitude obtained on a perfect reflector (aluminum foil placed between the slabs). All the measurements were performed statically in the middle of the slab. Results are shown in Fig. 7 (b), in which they are compared to numerical realistic simulations, performed for a reflection coefficient $R_{12}$ air-concrete of 0.45 . This coefficient was derived through eq. (1) from the wave speed, evaluated by dividing the double thickness of the slab by the travel time of the wave reflected on the bottom, and confirmed by the surface reflection method described in paragraph 2.2.

The linear dependence of the reflection coefficient on the thickness to wavelength ratio was still visible, even if for thicknesses less than $\lambda / 11$; some small discrepancies are observed. They could be due to some surface imperfections, or simply to the decrease of the signal to noise ratio. Anyway, we could show that layers with a thickness inferior to $\lambda / 100$ (about $1.3 \mathrm{~mm}$ here) could be visible with GPR. 


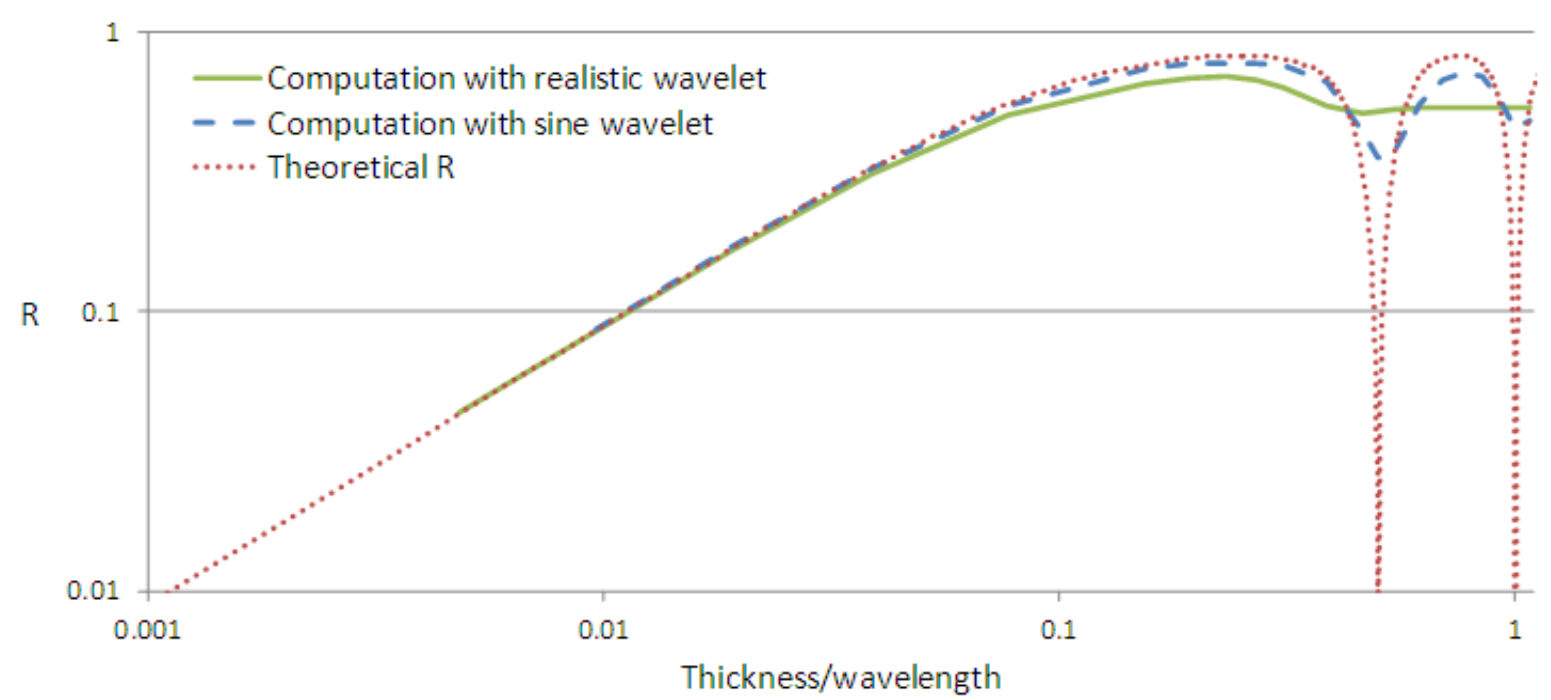

Figure 6: Reflection coefficient for an air thin layer imbedded at a depth of $10 \mathrm{~cm}$ into concrete $\left(\varepsilon_{r}^{\prime}=10\right)$, depending on the thickness on wavelength ratio. The theoretical estimation for a sine wave is compared to the modeled results obtained with a continuous sine wave and with a realistic short pulse.
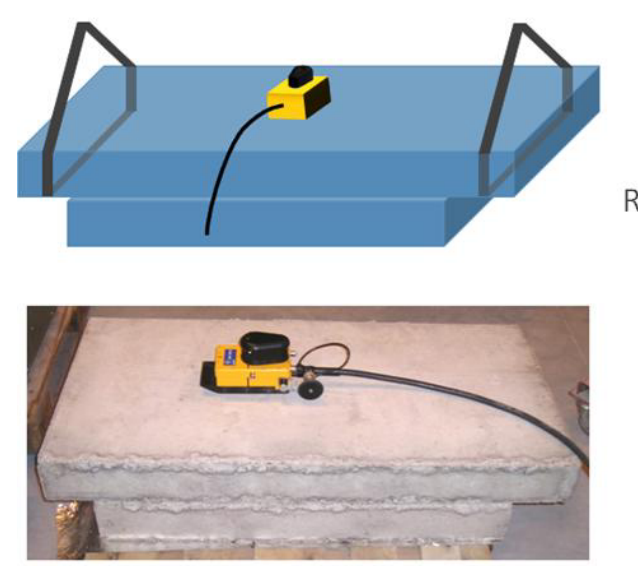

a

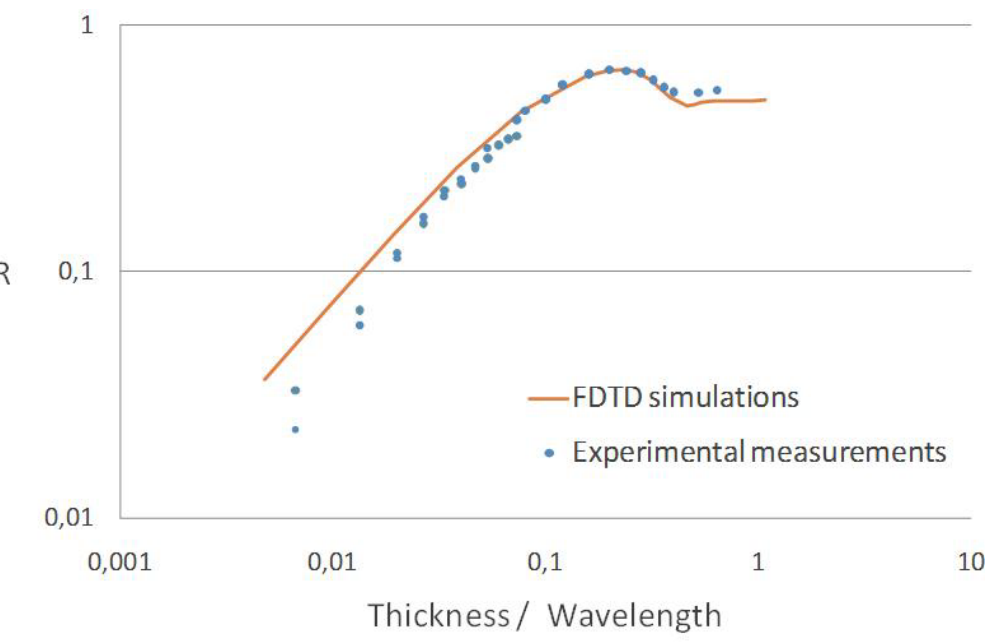

b

Figure 7: (a) Experimental setup; (b) Comparison between experimental measurements and numerical data.

\subsection{Influence of the Reflection Coeffi- cient on the Antenna Detection Limit}

The detection limit of the antenna is reached when the reflection amplitude from the thin layer is similar to the noise background level.

The reflection coefficient $R$ studied in this study is one of the parameters influencing the measured amplitude. It has been shown in the previous paragraphs that the parameters influencing directly this thin layer reflection coefficient are the layer thickness and the wavelength in the medium. The dominant wavelength $\lambda$ can be determined by eq. (6):

$$
\lambda=\frac{v}{f}
$$

In eq. (6), $v$ is the wave speed into the medium, and can be determined using eq. (1), while $f$ is the nominal frequency of the antenna.

The reflection coefficient $R$ has a linear influence on the amplitude measured at the surface, but other parameters influence this propagated signal. Eq. (7), modified from [8], describes the relationship between the amplitude measured by the radar $\left(\mathrm{A}_{\mathrm{obs}}\right)$ and the amplitude of the signal originally emitted by the antenna $\left(\mathrm{A}_{\text {source }}\right)$ :

$$
A_{\text {obs }}=C P \frac{e^{-\alpha 2 r}}{2 r} R A_{\text {source }}
$$


In eq. (7), $C$ and $P$ represent respectively the coupling factor with the ground and the radiation pattern for the emitter and receiver antennas, while $r$ is the depth of the thin layer and $\alpha(\mathrm{dB} / \mathrm{m})$ is the attenuation factor [2].

The coupling factor accounts for the energy loss at the antenna/ground interface and is highly influenced by the surface roughness. The radiation pattern is influenced by the antenna geometry, but also by the ground properties and by the surface contact quality. It is moreover frequency dependant, as well as distance dependant in the near field (at a distance inferior to about 8 wavelengths from the antenna) $[2,9]$. The factor $1 / 2 r$ accounts for the geometric spreading, while the factor $\mathrm{e}^{-\alpha 2 r}$ describes the intrinsic attenuation during the path into the matrix, caused by its conductivity [2]

From eq. (7), we see that for given matrix material and surface conditions, the measured amplitude and thus the detection limit depend on the depth of the layer as well as on its thickness and filling material.

The layer will be detectable if this measured amplitude is superior to the noise level. We distinguish the backscattering noise, caused by material heterogeneity and the white noise, inherent to the measurement precision of the antenna itself. The level of this last "white noise" can be lowered by using the stacking method, in which different measurements are performed at each point and then averaged, to eliminate the random noise.

\section{Conclusion}

In this study, we tried to evaluate the reflection coefficient of a thin layer into concrete, in order to estimate the detection limit of our antenna.

The reflection coefficient revealed to be linearly dependent on the thickness-on-wavelength ratio for thicknesses less than $\lambda / 11$; some small discrepancies were observed, probably due to the small surface imperfections. Anyway in our study, thicknesses inferior to $\lambda / 100$ were visible with the GPR.

We also showed that numerical simulation performed with a realistic impulsion and measured concrete properties could match real measurements very well and help to interpret experimental results.

By this method, the amplitude reflected by a thin layer can be estimated. The layer will then be visible if this reflected amplitude, after subtraction of the losses (including the intrinsic and geometric attenuations), is superior to the signal noise.

\section{Acknowledgements}

We thank the FNRS (National Found for Scientific Research in Belgium) for founding and Mr Lucien Dormal from Ronveaux S.A., who casted the smooth slabs.

\section{References}

1. I.L. Al-Qadi and S. Lahouar, Measuring layer thicknesses with GPR - Theory to practice, Construction and Building materials, 19(10) (2005) p. 763-772

2. A.P. Annan, Ground-Penetrating Radar, Near-Surface Geophysics Part 1: Concepts and Fundamentals, Society of Exploration Geophysicists, D. K. Butler (2005) pp. 357438

3. M.B. Widess, How thin is a thin bed? Geophysics, vol. 38 (1973) pp. 1176-1176

4. S. Lambot, A. Phuong Tran and F. André, Near-field modeling of radar antennas for wave propagation in layered media: when models represent reality, Proceedings of the 14th International Conference on Ground Penetrating Radar, Shanghai, China (June 4-8 2012)

5. J. Van der Kruk, R. Streich et al., Properties of surface waveguides derived from separate and joint inversion of dispersive TE and TM GPR data, Geophysics 71(1) (2006) K19-K29

6. J.W.S. Rayleigh, The Theory of Sound (2nd edn), vol. 2, Dover Publications, New York (1945)

7. A. Giannopoulos, Modelling Ground Penetrating Radar by GprMax, Construction and Building materials, vol. 19, no. 10 (2005) pp. $755-762$

8. J.H. Bradford and J.C. Deeds, Ground-penetrating radar theory and application of thin-bed offset-dependent reflectivity, Geophysics 71 (2006) p. K47-K47

9. Y.R. Jiao, G.A. McMechan, and E. Pettinelli In situ 2-D and 3-D measurements of radiation patterns of half-wave dipole GPR antennas, Journal of Applied Geophysics 43(1) (2000) p. 69-89 

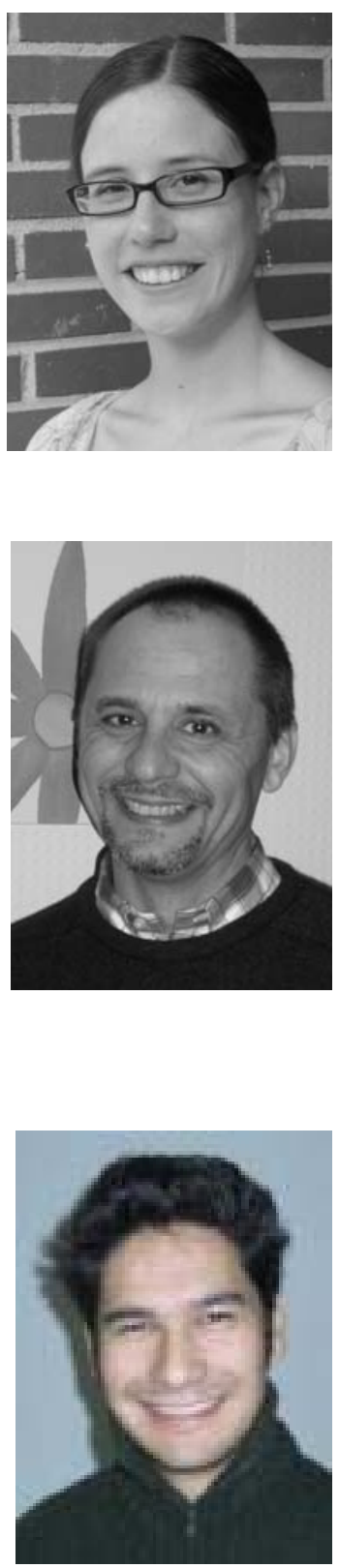

Received July 9, 2012
Audrey Van der Wielen, Ir. obtained in 2009 a Master Degree in Civil Engineering at the University of Liège, Belgium. She is currently a FNRS (national found for scientific research) research fellow and works on a Ph.D. thesis focusing on the use of ground penetrating radar for the detection of delaminations in concrete structures.

E-mail: avdwielen@ulg.ac.be

Luc Courard, Ph.D. is graduated from the University of Liège, Belgium where he obtained his $\mathrm{PhD}$ in 1998. He had a post-doctoral fellowship at Laval University, Québec, Canada where he developed the analysis of concrete surface and the effect of surface preparation techniques. He is also Honorary Professor at the Warsaw University of Technology. His research is focused on the study of adhesion of repair systems on concrete and the conditions of compatibility: surfometry analysis, physico-chemical interactions and effects of water have been considered as major parameters for research. Author or co-author of more than 100 publications in scientific journals and conference proceedings, he is also interested in waste and by-products recycling in civil engineering and durability estimation for reinforced concrete elements. He recently developed the use of vegetal fibers in insulating concrete panels and blocks. E-mail: Luc.Courard@ulg.ac.be

Frédéric Nguyen, Ph. D., is graduated from the University of Liège where he obtained his doctoral thesis in 2005. His main research interests are the development and application of geophysical methods in different contexts, with an aim to quantify the quality of information. He has contributed to the study of hydrogeological processes, particularly those related to saltwater intrusion and areas of preferential flow, of active faults in Western Europe. He also recently started working on the use of GPR for the characterization of concrete. Author or co-author of more than 30 publications in scientific journals and conference proceedings, he recently obtained a tenure track position at the University of Liège where he teaches geophysical and inversion methods in the Faculties of Applied Sciences and Sciences. E-mail: F.nguyen@ulg.ac.be 